\title{
La psychologie de la religion : courants de recherche et perspectives théoriques
}

Psychology of Religion: research trends and theoretical perspectives

\section{Magdalini Dargentas}

\section{(2) OpenEdition}

1 Journals

Édition électronique

URL : http://journals.openedition.org/assr/27216

DOI : $10.4000 /$ assr.27216

ISSN : $1777-5825$

Éditeur

Éditions de l'EHESS

\section{Édition imprimée}

Date de publication : 1 octobre 2015

Pagination : 25-44

ISBN : 978-2-7132-2515-4

ISSN : 0335-5985

Référence électronique

Magdalini Dargentas, "La psychologie de la religion : courants de recherche et perspectives théoriques ", Archives de sciences sociales des religions [En ligne], 172 | octobre-décembre, mis en ligne le 26 avril 2018, consulté le 19 avril 2019. URL : http://journals.openedition.org/assr/27216 ; DOI : 10.4000 /assr.27216 


\section{Magdalini Dargentas ${ }^{1}$}

\section{La psychologie de la religion : courants de recherche et perspectives théoriques}

Avec des contributeurs historiques, tels William James ou Stanley Hall, le religieux était l'un des premiers objets d'étude de la psychologie à la fin du XIX ${ }^{\mathrm{e}}$ siècle. William James, dont les écrits restent de référence et qui est souvent cité comme précurseur, a fortement influencé les travaux ultérieurs dans l'étude de la religion. La psychologie devenue discipline autonome, et les circonstances historiques amenèrent la quasi-extinction de la psychologie de la religion dans les années 1920. Elle se réduit alors à des considérations théologiques et philosophiques. Dans les années 1950, les études de la personnalité, et notamment celles de Gordon Allport sur la religiosité, relancent ce domaine. À partir des années 1970, la psychologie de la religion connaît un développement important surtout dans le monde anglo-saxon. Bien qu'elle soit riche de controverses, de nombreuses publications attestent son existence et son essor. À l'exception des travaux de Jean-Pierre Deconchy, récemment décédé, la psychologie française y est en retrait ${ }^{2}$.

L'étude qui suit propose un panorama des perspectives théoriques et empiriques majeures, depuis les années 1950, principalement dans le monde anglosaxon. Il s'agit de comprendre le développement et les orientations d'un domaine de recherche largement ignoré et peu développé en France. Notre lecture critique est nécessairement sélective ; elle s'en tient à une présentation des champs dominants. La présentation n'est pas chronologique, car les champs d'études, dont certains sont simultanés, sont trop imbriqués entre eux. Nous abordons : 1) les travaux de type "métrique », 2) ceux portant sur les corrélats de la religion, 3 ) les études sur la croyance, et 4) celles sur d'autres aires empiriques et théoriques du fait religieux ${ }^{3}$. Les critiques les plus fréquentes de ces travaux seront

1. L'auteur remercie Denise Jodelet, Françoise Dintinger, Ralph Balez et les experts anonymes pour leurs lectures critiques.

2. Signalons toutefois le récent numéro spécial des Assr, dirigé par Françoise Champion (2013), qui réunit un ensemble de contributions examinant les liens et les imbrications entre pratiques psychothérapeutiques et sphère religieuse. Pour un historique de la psychologie de la religion et une présentation de la situation en France, voir Dargentas (2016).

3. Nous utilisons l'expression "fait religieux " pour nous référer à toutes les formes de phénomènes liés à la religion (par exemple, développement religieux, conversion, mysticisme, attitude vis-à-vis de la religion, formes spécifiques des religions, religiosité, etc.). 
aussi évoquées. Enfin, nous soulevons des questionnements transversaux et relevons des tendances empiriques et théoriques qui illustrent des interrogations transversales dans ce domaine.

\section{Les travaux de type "métrique " issus de la psychologie de la personnalité}

À partir des années 1950, le paradigme dit "métrique » domine la psychologie de la religion: les diverses composantes de la religion, et notamment les attitudes religieuses, sont décrites au moyen de tests, inventaires et échelles. L'ensemble de ces travaux s'inscrit dans les théories de la personnalité. La religion y est pensée de manière multidimensionnelle. Gordon Allport est l'un des pères du paradigme «métrique ».

\section{La typologie de la religiosité de Gordon Allport et sa révision par Batson}

Gordon Allport mène ses travaux depuis les années 1920. Sa contribution est déterminante, dans la mesure où il a relancé la production scientifique en psychologie de la religion dans les années 1950 et parce que ses études inaugurent une tradition de recherche qui prévaudra jusqu'aux années 1980.

Allport s'inscrit dans une théorie nomothétique de la personnalité : la personnalité y est vue comme l'organisation des dispositions de l'individu, opérationnalisée et mesurée via des traits de personnalité (Allport, 1969, 1971).

Allport élabore la théorie de "l'Orientation Religieuse, Intrinsèque ou Extrinsèque ", opérationnalisée dans un ensemble d'échelles dites "ROS » (Allport \& Ross, 1967; Allport, 1969). Le développement de cette théorie est fondé sur les travaux étudiant les liens entre la religiosité et les attitudes sociales (notamment les préjugés). Allport s'appuie notamment sur les thèses d'Adorno sur la personnalité autoritaire associant religion et ethnocentrisme. Il se réfère aux conclusions de Rokeach montrant que les non-croyants sont moins dogmatiques et moins ethnocentriques. Il se rapporte également aux écrits de William James qui mettaient l'accent sur l'expérience religieuse individuelle et le rôle de l'émotion, en opposant le rapport personnel au rapport institutionnel à la religion. Allport s'inspire de cette distinction théorique, et élabore une typologie qui lui est propre : il oppose deux profils de religiosité (intrinsèque vs extrinsèque). La religiosité se réfère alors à la signification de la religion pour l'individu et sa manière de vivre la religion. Cette typologie lui permet de nuancer les assertions sur le lien entre religion et préjugés. Dans la théorie d'Allport, l'orientation intrinsèque est corrélée à la tolérance, alors que l'orientation extrinsèque est liée à l'ethnocentrisme ou au dogmatisme.

Dans l'orientation intrinsèque (I) la religion est une fin en soi, une valeur intériorisée par les individus; on «conçoit la foi comme une valeur suprême à 
part entière. Une telle foi s'efforce de transcender des besoins égo-centrés" (Allport, 1969 : p. 56) ${ }^{4}$. Cette orientation contribue à la "personnalité mature » définie par Allport (1971). Son opposée, l'orientation extrinsèque $(E)$, implique que la religion représente le moyen " instrumental » d'atteindre d'autres objectifs, de besoins identitaires et sociaux, "servant des motivations de confort personnel, de sécurité ou de statut social " (idem : p. 55). Outre ces deux orientations, Allport et Ross (1967) ont défini les « indiscriminate proreligious » qui s'élèvent sur les deux échelles, intrinsèque et extrinsèque. Ils ont aussi défini les "indiscriminate antireligious » ou «nonreligious » qui expriment leur désaccord sur ces deux échelles, et qui concernent donc les non religieux. Ces deux dernières orientations sont généralement oubliées par les chercheurs en faveur d'une théorie bipolaire opposant dimensions extrinsèque et intrinsèque.

Donahue (1985) dresse la revue critique la plus complète des travaux en question. Batson révise le modèle d'Allport, en 1976, puis en 1982 (Batson, Schoenrade, \& Pych, 1985). Il approfondit la distinction d'Allport entre religiosité mature et immature. Pour Batson, la religiosité mature se caractérise par le doute et l'autocritique, caractéristiques qui figurent dans la théorie initiale d'Allport mais que l'orientation «I » ignore. Le «Religious Life Inventory » de Batson adjoint à la théorie initiale d'Allport une troisième orientation, nommée " quest» (ou «interactional scale»). Elle concerne la religiosité non alignée sur une institution religieuse et avec une forte tendance à s'interroger sur la vie et/ou la structure sociale.

La principale critique de ces théories sur la religiosité repose sur l'implication idéologique de ses auteurs. Selon Beit-Hallahmi (1985 : p. 25), «Allport est proreligieux [...] se pose comme un défenseur du protestantisme libéral qu'il oppose au protestantisme conservateur ". La distinction des orientations extrinsèque et intrinsèque serait une tentative de défendre les effets positifs de la religion face aux travaux associant la religiosité au recours aux préjugés. Batson, théologien et psychologue, serait aussi influencé par son propre système de croyances. La dimension "quest", envisagée positivement, semble refléter sa propre identité de protestant (Belzen, 1999). Ces critiques n'occultent en rien la richesse des travaux sur la religiosité ; elles permettent toutefois de les resituer dans leur contexte de production.

\section{D'autres travaux métriques : l'orthodoxie, le fondamentalisme, manières d'interpréter les croyances}

D'autres tentatives pour établir des typologies à propos de l'orientation religieuse ont également vu le jour.

4. Toutes les citations données dans notre article sont traduites par nos soins de l'anglais. 
Les mesures de l' " orthodoxie chrétienne » évaluent l'adhésion déclarée aux doctrines chrétiennes. Certains s'appuient sur la distinction de Lenski dans les années 1960 opposant l' " orthodoxie doctrinale » au " dévotionalisme ». L'échelle de "Christian Orthodoxy» (Fullerton \& Hunsberger, 1982; rapportés par Hunsberger, 1991) est son prolongement le plus récent.

Certains tentent de mesurer le fondamentalisme : par exemple, la «Religious Fundamentalism scale " dépasse le cadre chrétien pour mesurer la croyance en une réalité absolue (Altemeyer \& Hunsberger, 1992). Cette échelle, testée dans plusieurs cultures et auprès de divers groupes religieux, montre que l'adhésion relative à une "réalité absolue » transcende les appartenances religieuses et les communautés de croyants.

À propos de l'interprétation de la religion, et de la manière de donner du sens aux croyances religieuses, Hunt (1993) met en place les "LAM scales" pour étudier les dimensions "Littérale », "Anti-Littérale » et "Mythologique » (ou «symbolique»). La dimension littérale implique l'adhésion à une doctrine, de manière fondamentaliste. Dans la dimension «mythologique " on interprète la doctrine de manière symbolique. La dimension "anti-littérale " implique le rejet total de la doctrine. Enfin, la «Post-Critical Belief scale » de Hutsebaut (1996 ; rapporté par Fontaine, Duriez, Luyten, \& Hutsebaut, 2003) suggère une approche bidimensionnelle de la religion et vise l'étude des différences interindividuelles dans un contexte chrétien. La première dimension porte sur l' «Inclusion » ou l' «Exclusion » de la "Transcendance », tandis que la seconde oppose les interprétations «Littérale " et «Symbolique » de la religion. Quatre attitudes envers la religion sont alors déterminées :

a) l' «Orthodoxie » dans l'intersection des dimensions « Littérale » et « Inclusion de la Transcendance ", concernerait les fondamentalistes. b) La "Critique Externe », située entre les dimensions « Littérale » et « Exclusion de la Transcendance ", implique le rejet de la religion dans une attitude antireligieuse. c) Le "Relativisme" au croisement des dimensions «Symbolique » et « Exclusion de la Transcendance » : bien qu'il admette le sens caché des mythes et rituels de la religion, l'individu rejette la religion. d) La "Naïveté seconde ", constituée des dimensions "Symbolique » et "Inclusion de la Transcendance ", serait une affirmation de l'existence de la religion, l'individu tenterait d'interpréter le langage religieux de manière symbolique.

Mentionnons un dernier type de travaux qui tente d'identifier des dimensions communes à toutes les religions. Citons la typologie de Glock et Starck dans les années 1960, qui compte cinq dimensions : idéologique, ritualiste, expérientielle, intellectuelle et conséquentielle. Cette typologie est à l'origine de nombreux travaux mobilisant des "5-D scales" (5-Dimensions scales). Cependant, Clayton et Gladden (1974) montrent qu'en dépit du projet universalisant de Glock et Stark, visant à identifier des dimensions communes à toutes les religions, leur conception est très influencée par le christianisme. 
Le «Structure-of-Religiosity Test»(S-R-T) de Huber (1996, rapporté par Huber, 2006) est un prolongement récent de cette approche. Huber évoque les dimensions " intellectuelle ", "idéologique », " dévotionnelle ", " expérientielle » et la "dimension de la pratique religieuse publique » en y intégrant également la «centralité » et le "contenu » de la religiosité. La "centralité » se rapporte à l'importance de la religion pour un individu et son impact sur son comportement ; le «contenu » renvoie aux croyances, attitudes ou orientations de la religiosité. Le « S-R-T » a été validé dans 12 pays sans se limiter au seul christianisme.

\section{Les corrélats de la religion}

Autre volet du paradigme «métrique », les corrélats de la religion abordent spécifiquement le comportement ou la personnalité. En essor depuis les années 1980, on y étudie par exemple les attitudes sociales, les comportements moraux en société, la santé, voire les traits de personnalité associés. Toutefois, rappelons qu'il s'agit d'un champ d'études déjà existant dans les années 1950. Comme il a été dit plus haut, Allport a conçu sa typologie dans l'objectif de préciser les liens entre les profils de religiosité et le recours aux préjugés. Ainsi, dans ces travaux, les typologies de la religiosité d'Allport et Batson sont souvent utilisées.

\section{Les attitudes sociales}

Depuis Allport et Batson, on étudie souvent les attitudes ethnocentriques, conservatrices et discriminatoires liées à des dispositions proreligieuses. On met souvent en évidence une relation positive entre une pratique religieuse et une attitude ou des préjugés racistes (voir : Argyle, 1985, 2002 ; Batson et al., 1985 ; Beit-Hallahmi \& Argyle, 1997 ; Brown, 1985 ; Dittes, 1969 ; Donahue, 1985 ; Gorsuch, 1988 ; Gorsuch \& Aleshire, 1974).

De même, nous l'avons vu aussi, les liens entre les attitudes ethnocentriques ou discriminatoires et l'appartenance à une religion dépendent du type de religiosité. Les « intrinsèques » ont moins de préjugés que les « extrinsèques » (Allport, 1969). Cependant, une relation curvilinéaire semble prévaloir : les personnes très religieuses et les personnes non religieuses auraient le moins de préjugés (Gorsuch \& Aleshire, 1974).

Ce résultat est néanmoins révisé : car on y relève des résultats contradictoires et des artefacts liés à la désirabilité sociale (Batson, Schoenrade, \& Pych, 1985). Les «intrinsèques " rapporteraient moins de préjugés et d'attitudes discriminatoires pour paraître plus tolérants. L'orientation «quest» ferait état de moins de préjugés que les extrinsèques et les intrinsèques (idem).

Dans cet ensemble d'études, plusieurs explications sont proposées : par exemple, la soumission idéologique à une institution religieuse enlèverait à l'individu son indépendance de jugement, ou encore l'affiliation à un groupe religieux instaurerait une distance sociale à l'égard d'autres groupes. 
Parmi les critiques adressées à ce champ d'études, on trouve la difficulté d'interpréter le lien entre les attitudes sociales et l'engagement religieux (Gorsuch, 1988). Il semble que d'autres variables (sexe, âge, niveau scolaire) peuvent influencer les résultats. Enfin, les indicateurs choisis pour mesurer la religiosité ou les attitudes sociales, généralement très différents d'une recherche à l'autre sont souvent mis en cause (Gorsuch \& Aleshire, 1974).

\section{Les comportements moraux et prosociaux}

Concernant la religiosité et les comportements prosociaux, moraux ou altruistes, les hypothèses scientifiques s'étayent sur le sens commun : à savoir, l'attachement à une religion sensibiliserait aux besoins d'autrui par adhésion aux valeurs morales.

Ici, les indicateurs du comportement prosocial ou altruiste sont : l'empathie, le volontariat, la charité, la suspicion ou la confiance accordée à autrui. Les personnes religieuses adopteraient plus de comportements d'entraide que les non religieux (voir Argyle, 2002 ; Beit-Hallahmi \& Argyle, 1997 ; Batson et al., 1985). Cependant, les adeptes fondamentalistes ou conservateurs seraient plus suspicieux et moins altruistes en fonction de l'appartenance de la cible. Une étude illustrant cet effet négatif montre que les homosexuels et les mères célibataires sont tenus pour responsables de leur chômage par des personnes ayant un score élevé de fondamentalisme (Jackson \& Esses, 1997).

Les intrinsèques seraient plus dévoués à l'autre que les extrinsèques; ils auraient davantage le souci d'apparaître compatissants et agiraient de façon plus automatique, selon des scripts, sans être cependant à l'écoute réelle d'autrui (Batson et al., 1985); ils chercheraient à être reconnus comme altruistes (Jackson \& Esses, 1997). L'orientation « quest » impliquerait un réel comportement altruiste défini par le respect des besoins réels d'autrui : on y respecte par exemple le refus de se faire aider.

Dans un cadre prosocial toujours, d'autres travaux de psychologie de la religion envisagent explicitement l'étude des vertus comme un terrain idéal pour appréhender les aspects positifs de l'individu (Emmons \& Paloutzian, 2003 ; Hill, 1999). Martin Seligman (président de l'Association américaine de psychologie - APA), dans son discours au congrès de l'APA (1998; Seligman, 1999), évoque la psychologie comme une science humaine positive dont l'intérêt est d'aborder les côtés positifs de la vie, avec des thèmes comme la force personnelle ou la vertu civique. L'espoir, l'humilité, l'honnêteté, la gratitude, la sagesse, le contrôle de soi, le pardon comme objets universels et socialement nécessaires, avec un impact sur la santé mentale et le bonheur, attirent donc les chercheurs. Ceci relève d'une conception idéologique de l'humain qui conduit à négliger d'autres qualités ( $p$. ex., autonomie, indépendance, courage, singularité, etc.). 
Nous rejoignons ainsi les critiques précédemment évoquées ( $c f$. Belzen, 2005a ; Hill, 1999) autour des conceptions instrumentalisées de la religion, exclusivement focalisées sur ses effets positifs, et qui peuvent dissimuler un arrière-plan idéologique et politique.

\section{La religiosité immunogène ?}

Plusieurs travaux étudient le lien entre la religion, la santé physique ou mentale et le bien-être des individus. Les personnes " religieuses » seraient en meilleure santé que les personnes non religieuses et auraient une meilleure espérance de vie. Les explications avancées renvoient aux habitudes de vie spécifiques (immunogènes). Dans ces études, les personnes religieuses fument et boivent moins, se déclarent en meilleure santé physique et mentale, expriment un bien-être subjectif plus important et une moindre peur de la mort comparés aux non-croyants. Toujours selon ces travaux, les pratiquants, comparés aux non pratiquants, ont moins de troubles cardiaques, sont moins dépressifs, ils éprouvent moins de sentiments de culpabilité, d'anxiété, ou d'agressivité ; le risque suicidaire est également plus faible chez les pratiquants. Ils se déclarent plus satisfaits de leur vie, plus heureux, gèrent mieux un deuil, croient plus à une vie après-mort, et ont moins peur de la mort. Ces résultats sont expliqués par le rôle positif des croyances religieuses, mais aussi par le support social dont les pratiquants peuvent bénéficier (voir Argyle, 1985, 2002 ; Beit-Hallahmi \& Argyle, 1997 ; Donahue, 1985 ; Gorsuch, 1988).

Des relations différentes sont aussi envisagées en fonction des types de religiosité : la religiosité intrinsèque impliquerait une meilleure santé physique et mentale que l'orientation extrinsèque (voir le travail de Ryan, Rigby et King, 1993).

Ce courant de recherche apporte des données très intéressantes, mais l'opérationnalisation des concepts et méthodes est critiquable ; de même, la généralisation abusive des résultats, parfois contradictoires, y est habituelle.

La critique récurrente reste l'instrumentalisation idéologique de ces recherches du fait de l'appartenance spirituelle des chercheurs souhaitant prouver l'influence bénéfique de la religion (Belzen, 2005a ; Hill, 1999).

\section{Les traits de personnalité}

Un champ d'études aborde le lien entre la religion et les types ou traits de personnalité. On a ainsi associé la religiosité et l'estime de soi en envisageant une personnalité de type « dépendant » avec un « ego faible ». Toutefois, un lien positif entre la religiosité et une estime de soi positive a été aussi identifié (voir Dittes, 1969 ; Beit-Hallahmi \& Argyle, 1997). Le plus souvent, ces études recourent à deux types de tests: le "Eysenck Personality Inventory» (EPI) et le "Five Factor Model » (connu sous le nom de «Big Five Questionnaire ») ou son successeur, le NEO-PI-R (Neuroticism, Extraversion, Openness, Personality InventoryRevised). 
Selon certaines études descriptives utilisant le EPI ${ }^{5}$, les femmes sont plus religieuses que les hommes, les introvertis plus religieux que les extravertis. De même, les personnes religieuses s'élèvent davantage dans l'échelle du mensonge que les autres (Francis, 1985). Le psychotisme serait négativement corrélé avec la religiosité, alors que la pensée magique (p. ex., croyance en la télépathie) serait liée à des expériences de type schizoïde (Beit-Hallahmi \& Argyle, 1997).

Les études utilisant le Big Five ou le NEO-PI-R ${ }^{6}$, observent que le "névrosisme » et l'extraversion sont indépendants de la religiosité. Une revue métaanalytique montre un lien entre religiosité, " agréabilité » et " esprit consciencieux » (Saroglou, 2002).

Ces études sur les traits de personnalité sont intéressantes, mais les liens entre la religiosité et les dimensions caractérisant la personnalité de l'individu doivent être précisés. Certes, cette difficulté est récurrente dans le champ métrique. Cela illustre à notre sens les limites de ce paradigme, et la difficulté de restreindre par nécessité un objet complexe comme la personnalité ou la religiosité à quelques variables sélectives et partielles.

Abordons maintenant un autre champ d'études, celui de la croyance examinée d'un point de vue développemental.

\section{Travaux sur la croyance et le développement de la personne}

Les recherches centrées sur la croyance, envisagée du point de vue de son développement s'appuient sur des théories cognitives, de la socialisation et psychodynamiques en étudiant, par exemple, l'image de Dieu chez les enfants.

Parmi les théories à orientation cognitive et développementale, de caractère structuraliste et néo-piagetien, nous pouvons citer le modèle du théologien James Fowler (1981 ; rapporté par Fowler, 1994), nommé « Faith Development Theory ». Il définit des stades universels, hiérarchisés et irréversibles du développement de la foi. Fowler s'inspire des travaux de Piaget des années 1930 et sur ceux de Kohlberg dans les années 1960 portant sur le développement moral.

Fowler s'intéresse plus à la structure et au type de raisonnement qu'au contenu associé à chaque stade. Les stades vont en se complexifiant et sont indépendants de toute tradition religieuse. Chaque stade se caractérise par un certain rapport de l'individu aux croyances (Fowler, 1994).

5. Ce test comprend quatre dimensions : introversion-extraversion; stabilité-névrosisme ; échelle du mensonge mesurant le conformisme social ; et " psychotisme " (absence de sentiments de compassion ou d'empathie).

6. Ce test comprend cinq dimensions: névrosisme (stabilité vs instabilité émotionnelle), extraversion ( $v s$ introversion), ouverture à l'expérience ( $v s$ conformisme), agréabilité ( $v s$ antagonisme), caractère consciencieux ( $v s$ impulsif). 
Le premier stade (1-intuitive-projective faith) se caractérise par l'égocentrisme et l'imagination, et une conscience intuitive des mystères de la vie. Le deuxième stade (2-mythic-literal faith) est porté par l'interprétation littérale des croyances religieuses, une morale de type punition-récompense et l'adhésion aux croyances de la cellule familiale. Le troisième stade (3-synthetic-conventional faith) connaît une influence du groupe social élargi dépassant la seule aire familiale ( $p$. ex., école, amis, médias, etc.) ; ce stade est marqué par le conformisme social et l'absence de raisonnement critique. Le quatrième stade (4-individuative-reflective faith) implique un raisonnement critique sur les croyances adoptées qui se retrouvent démythifiées; la foi est de type idiosyncrasique. Le cinquième stade (5-conjunctive faith/ paradoxical-consolidative faith) opère une intégration de visions qui paraissaient inconciliables au départ et une redécouverte des croyances. Le dernier stade (6-universalizing faith), que peu d'individus peuvent atteindre selon la théorie de Fowler, implique l'adoption de valeurs universelles transversales.

Ce modèle théorique soulève nombre de débats. Les critiques mettent en cause le caractère universel, invariant, statique et l'organisation hiérarchique des stades proposés. On oppose au modèle une alternative constructionniste qui s'inscrit dans une perspective discursive ou narrative. L'accent est mis sur les réseaux de discours et leur influence sur la communication et les conceptions du monde par les individus (Day, 2001). Dans cette perspective, l'expression des croyances s'inscrit dans des contextes relationnels où elles ont des fonctions précises (Gergen, 1993).

Comme alternative, le «Religious Styles Perspective » (RSP) de Streib (2001) revisite le modèle de Fowler en tenant compte de ses critiques; il y introduit le vécu du sujet. Des théories phénoménologiques et psychodynamiques y sont mobilisées qui mettent l'accent sur l'émotion, le rapport à autrui, et la pragmatique de la communication. Streib conteste le principe de stades invariants et hiérarchisés et propose de parler des «styles religieux » qui seraient des modes rituels, symboliques et narratifs d'appropriation de la religion. Ceux-ci dépendraient du développement du sujet, mais surtout de son histoire personnelle. Streib définit cinq styles : 1) le style religieux subjectif (subjective religious style) qui correspond au premier stade de Fowler ; 2) le style instrumental-réciproque (instrumental-reciprocical or "do-ut-des » religious style) où l'enfant, conscient de ses besoins, entre dans une relation avec Dieu comme autrui selon un schéma punition/récompense ; 3 ) le style religieux mutuel (mutual religious style) correspond à des relations de réciprocité basées sur l'amour, le respect et la sécurité du groupe religieux d'appartenance ; 4) le style religieux individualisant-systémique (individuative-systemic religious style) est dominé par la réflexion, le scepticisme autour du religieux et un raisonnement critique ; 5) le style religieux dialogique (dialogical religious style) véhicule l'intégration de contradictions et l'ouverture envers les individus ayant des orientations religieuses différentes. 
Pour conclure ici, Rizzuto (2001) discute ces approches et défend la nécessité d'une théorie du développement de la foi multidimensionnelle, imbriquant à la fois les acquis psychanalytiques, les styles interpersonnels et les perspectives narratives.

L'étude de la croyance, envisagée de manière développementale, s'inscrit aussi dans des recherches portant sur la socialisation; on examine l'impact de l'entourage social (famille, pairs, etc.) sur sa religiosité.

Selon ces approches, le contexte familial influence la religiosité, les femmes tendent à être plus religieuses que les hommes, les mères transmettent plus que les pères les valeurs religieuses, un enseignement estimé excessif de la religion provoque un comportement d'opposition. La question de la conversion est également étudiée; elle semble survenir entre 12 et 17 ans en lien avec des crises émotionnelles adolescentes (voir Beit-Hallahmi \& Argyle, 1997 ; Gorsuch, 1988 ; Hunsberger, 1991). De même, le rejet de l'affiliation religieuse par l'individu s'avère inextricablement lié aux relations familiales ; c'est l'étude de l' " apostasie » (Hunsberger, 1980).

Concernant l'image de Dieu chez le croyant, une première orientation empirique mobilise les théories psychodynamiques d'inspiration freudienne. Les analyses portent sur la projection, en examinant le lien entre les figures parentales et la formation du concept ou de la représentation de Dieu. On met aussi l'accent sur la dynamique des réponses des sujets et on y examine des processus inconscients, des aspects émotionnels et des représentations qui conditionnent les réponses des sujets (Rizzuto, 2001 ; Vergote \& Tamayo, 1981). L'étude de la formation du concept de Dieu a reçu également la contribution de Deconchy (1967), montrant que l'idée de Dieu, pour des enfants ayant entre 8 et 16 ans, est davantage associée à des images maternelles chez les garçons, et paternelles chez les filles.

Une deuxième orientation empirique s'appuie sur la théorie de l'attachement de Bowlby, on y examine l'attachement de l'individu à la divinité. Ici, la relation avec Dieu est influencée par les expériences du jeune enfant (Kirkpatrick, 1999). Ces recherches souvent expérimentales s'inscrivent généralement dans un contexte culturel chrétien ou juif; la validité de ce champ d'études pourrait être améliorée par le recours à d'autres populations et par la mise en place de recherches de type longitudinal.

\section{D'autres apports théoriques autour du fait religieux}

Sont examinés ici des travaux sur la spiritualité, ainsi que certaines contributions des sous-disciplines de la psychologie.

\section{Objets empiriques : formes d'expériences religieuses et spirituelles}

La psychologie de la religion aborde aussi des formes d'expériences religieuses et spirituelles comme les pratiques religieuses traditionnelles qui reprennent 
du terrain ( $p$. ex., la méditation, Van der Lans, 1985), les «nouveaux mouvements religieux » en référence aux cultes et phénomènes se détachant des formes religieuses traditionnelles (Buxant, Saroglou, Casalfiore, \& Christians, 2007), l'athéisme (Vergote, 1996), les sujets occultes (magie, astrologie, croyances autour du paranormal, Beck \& Miller, 2001).

Ces objets illustrent l'intérêt pour l'étude de la spiritualité avec une orientation théorique tournée vers l'approche des émotions. Les phénomènes religieux seraient en transformation et les pratiques d'ordre spirituel en augmentation (Argyle, 2002). La spiritualité semble ainsi en vogue parmi les chercheurs qui la distinguent généralement de la religion : la notion mobilise davantage l'expérience personnelle et des valeurs individualistes (Emmons \& Paloutzian, 2005 ; Peet, 2005). Ceci dit, l'opposition entre religion et spiritualité est discutable (Zinnbauer, Pargament, \& Scott, 1999) car les formes institutionnelles de la religion comportent des dimensions spirituelles tandis que certains phénomènes à dimension spirituelle s'accompagnent d'organisation sociale ( $p$. ex., groupes constitués autour des croyances New Age, du yoga ou des sites web à contenu spirituel). Ces formes religieuses et spirituelles sont une occasion idéale pour l'étude des affects et des émotions (Emmons \& Paloutzian, 2003 ; Hill, 1999). La religion y est étudiée en tant qu'expérience émotionnelle intense ( $p$. ex., Hood, 1980).

\section{Autour du fait religieux : neurosciences, cognitions et psychologie sociale}

Les neurosciences. La neuropsychologie s'intéresse à certains phénomènes spirituels; le terme de "neurothéologie » désigne la contribution de ce champ à la connaissance de la religion. On y examine les corrélats neurophysiologiques des expériences religieuses et mystiques ; ceci est toujours lié à l'étude des émotions. Deux préoccupations empiriques dominent : l'induction des expériences mystiques et l'activité neurophysiologique associée aux expériences comme la méditation ou la prière (Kirkpatrick, 1999).

La première perspective étudie le rôle des substances exogènes ( $p$. ex., LSD, mescaline) et endogènes ( $p$. ex., les endorphines liées aux états de stress et de douleur), ou celui des techniques physiques (hypoventilation, hyperventilation) sur l'induction d'expériences similaires à des expériences mystiques. Cependant, loin d'assimiler ces expériences à des expériences mystiques, leur interprétation par l'individu semble dépendre du contexte dans lequel il les éprouve (Argyle, 1985 ; Dittes, 1969 ; Hay, 1985).

La deuxième perspective aborde les acquis des études de l'activité du cerveau, qu'on désigne en tant que brain research. On y utilise des outils comme l'électroencéphalogramme, la tomographie spectrale, ou la résonance magnétique. Ces investigations montrent que la méditation et la prière induisent des altérations de l'activité électrochimique du cerveau et que l'hémisphère gauche tend à diminuer 
significativement son métabolisme (Hay, 1985). Des travaux plus récents montrent que le lobe pariétal (participant à l'orientation spatiale) diminue son activité lors d'expériences mystiques (Newberg \& D'Aquili, 2000). Ces travaux font néanmoins l'objet de critiques d'ordre méthodologique - biais d'échantillonnage - et épistémologique - difficulté de définir l'objet d'études (Aletti, 2006).

Les approches cognitives. Les approches cognitives, quant à elles, étudient la mémoire ou la vitesse de jugement auprès des populations croyantes. La littérature se réfère généralement à trois champs (voir pour une revue Van der Lans, 1977) : 1) La théorie de l'attribution, étudiant les processus de codage et d'attribution de sens ainsi que leurs biais en lien avec les expériences religieuses. 2) Les cadres de référence religieux, expliquant que le fait d'être familier avec les symboles et les mythes d'une tradition religieuse est une condition de l'expérience religieuse (voir l'école suédoise des années 1960, et notamment les travaux de Sundén et de ses élèves, in Van der Lans, 1985). 3) Enfin, le modèle de traitement d'informations (information-processing model) analyse les facteurs sous-jacents à l'expérience religieuse en se focalisant sur l'activation de schèmes de mémoire, rendant possible l'interprétation d'une situation comme étant de type religieux. Une attention particulière est portée à la perception altérée suite à de fortes émotions; on y recherche les processus qui activent une expérience religieuse (crise nerveuse ou psychologique).

Enfin, la croyance religieuse est étudiée selon une perspective évolutionniste. Cette approche exploite les acquis de l'anthropologie cognitive et des neurosciences (Boyer, 2003 ; Kirkpatrick, 1999). Selon Boyer, les systèmes mentaux mobilisés par le fait religieux fonctionnent également en contexte non religieux. Propres à l'évolution de l'homme, ils répondent à différents besoins : échanger avec les autres, comprendre le monde, répondre à la peur de la mort et du malheur. Boyer souligne que le caractère non rationnel des croyances religieuses est une constante universelle des différentes formes religieuses.

Approches psychosociales. La religion a été un objet d'études princeps de la psychologie sociale, sans que cette discipline revendique une affiliation à la psychologie de la religion ${ }^{7}$. Par exemple, l'ouvrage à l'origine du développement de la théorie de la dissonance cognitive de Festinger, Riecken et Schachter (1956) s'étaye sur l'étude par observation participante d'un groupe sectaire. "L'échec d'une prophétie » retrace les transformations des croyances de ce groupe dont

7. Ceci est dû à la réticence des chercheurs en psychologie à inclure le fait religieux parmi leurs objets d'étude. Cette réticence peut-être expliquée par des facteurs qui ne sont pas l'objet du présent article (critique des motivations des chercheurs, objet considéré comme relevant de la philosophie, difficulté d'appliquer une démarche scientifique, etc.). Par conséquent, dans l'analyse de phénomènes religieux, les chercheurs ont tendance à les utiliser comme contextes empiriques pour l'élaboration des théories plus générales (ici, la dissonance cognitive) et n'établissent pas des liens avec la psychologie de la religion. 
la prophétie qui annonce la fin du monde s'avère invalide. Ce livre raconte que la force de l'engagement dans une croyance profonde, le soutien social et l'inconfort (ou la dissonance) que procure le caractère finalement erroné des croyances conduisent certains individus à adopter d'autres stratégies que l'abandon rationnel de ces croyances ( $p$. ex., prosélytisme). Les approches psychosociales apparaissent également dans d'autres domaines déjà évoqués (corrélats comportementaux de la religion, et attribution causale par exemple) mais aussi dans un mode de régulation à la fois personnel et social : l'orthodoxie idéologique (Deconchy, 1995).

Selon l'orthodoxie idéologique, un individu est orthodoxe quand sa pensée, son langage et son comportement sont régis par le groupe idéologique dont il fait partie. Deconchy (1971) se focalise sur les structures qui organisent les contenus individuels et groupaux des croyances. Il effectue ses travaux expérimentaux au sein de l'Église catholique romaine. Deconchy (1998) affirme que son parcours de chercheur est lié à son intégration dans l'Église catholique, où il était difficile de penser les croyances autrement.

D'autres théories psychosociales sont également utilisées pour analyser le fait religieux : la soumission idéologique, la distance sociale, le management de la terreur. À cela s'ajoutent des processus intra et hors groupe, le statut minoritaire ou majoritaire d'un groupe religieux, la croyance en un monde juste, le locus of control, la communication verbale et non verbale (cf. Argyle, 1985, 1988, 2002 ; Beit-Hallahmi \& Argyle, 1997 ; Brown, 1985 ; Dittes, 1969 ; Jackson \& Esses, 1997 ; Gorsuch, 1988).

Enfin, des travaux plus récents (Dargentas, 2005 ; Dargentas \& Fraïssé, 2011) prennent comme cadre théorique les représentations sociales, ce qui permet de rejoindre certaines perspectives culturelles en psychologie de la religion (Belzen, 1999, 2005b). Centrée sur la construction du sens, la théorie des représentations sociales (Jodelet, 1989) permet d'étudier les significations attribuées aux phénomènes religieux, leur construction, les mécanismes liés, ainsi que leur inscription dans la dynamique intra-individuelle, interindividuelle, groupale, culturelle et enfin historique. Elle permet aussi d'étudier le fait religieux à partir des contenus que les individus empruntent pour défendre des points de vue à propos d'objets sociaux controversés non directement liés. Dargentas (2005) et Dargentas et Fraïssé (2011) montrent l'émergence des contenus religieux, dans certaines problématiques sociales (comme la crémation ou le mariage homosexuel dans des contextes conflictuels), sans qu'ils soient nécessairement liés à des acteurs religieux. Les contenus religieux apparaissent alors comme des " routines de pensée ». Une telle approche permet d'élargir la définition des phénomènes religieux susceptibles d'intéresser la psychologie de la religion pour y inclure des contenus religieux qui sont spontanément mobilisés pour aborder les objets sociaux. 


\section{Pour conclure : un domaine en crise et en transformation}

La description des différents courants théoriques et empiriques précédents met en évidence l'implication idéologique des chercheurs dans l'étude du fait religieux. Cette implication peut les conduire à légitimer, de façon assumée ou non, leurs propres systèmes de croyances. Cela nuit à la qualité des travaux et à la compréhension scientifique du religieux. Cette tendance se manifeste notamment par le choix de certains objets ( $p$. ex., la religiosité et de ses corrélats), par les conceptions des formes religieuses estimées positives ou négatives ( $p$. ex., la religion envisagée négativement versus la spiritualité envisagée positivement), par l'attitude pro ou antireligieuse ou encore par la référence à l'histoire personnelle du chercheur (Belzen, 1999 ; Beit-Hallahmi, 1985 ; Deconchy, 1987 ; Ragan, Malony, \& Beit-Hallahmi, 1980 ; Vergote, 1996 ; Zinnbauer et al., 1999). Le risque est de faire une apologie du fait religieux, passant d'une psychologie de la religion à une " psychologie religieuse " parfois revendiquée, ou bien de rejeter l'étude du religieux en raison de l' "impossible neutralité » dans la posture de recherche, ce qui a été principalement le cas en psychologie.

Au niveau méthodologique, les problèmes persistent : la faiblesse dans l'opérationnalisation des concepts et de la validité écologique, les résultats contradictoires dans un champ empirique ( $p$. ex., les corrélats de la religiosité), la focalisation sur des échantillons chrétiens (généralement étasuniens), la difficulté de conclure en termes de causalité, l'omission de variables susceptibles d'influencer les résultats (Gorsuch, 1988 ; Hunsberger, 1991 ; McFarland, 1984 ; Vande Kemp, 1999). De même, les méthodes expérimentales sont insuffisamment maîtrisées (Batson, 1979 ; Deconchy, 1985 ; Yeatts \& Asher, 1979), comme les méthodes qualitatives (Aten \& Hernandez, 2005 ; Belzen, 2005b ; Vergote, 1993).

Sur un plan théorique, la diversité des courants et des démarches empiriques peut donner l'impression que la psychologie de la religion constitue un domaine " éparpillé » avec des conceptions hétérogènes. On note ainsi la transformation des conceptions même de la religion, intégrant les nouveaux "faits spirituels ", dans les analyses des chercheurs (Belzen, 1999, 2005a ; Peet, 2005 ; Zinnbauer et al., 1999). L'adoption des concepts ou théories de la psychologie générale, et leur simple transfert vers l'étude du fait religieux sont contestés; la psychologie de la religion aurait besoin de développer ses propres théories adaptées à ses propres objets d'étude (Belzen \& Hood, 2006). Cette emprise épistémologique de la psychologie sur la psychologie de la religion est parfois considérée comme un facteur limitant son développement. Ainsi, ces controverses font que le domaine semble en crise : il se questionne et recherche son identité et son unité (Belzen, 1999; 2005b ; Nørager, 1996). Toutefois, cela constitue aussi son dynamisme et sa richesse.

Deux orientations sont suggérées par les chercheurs pour l'avenir de la psychologie de la religion. Elles ne sont pas nouvelles, mais elles ont été longtemps considérées comme incompatibles par leurs défenseurs. Elles se présentent maintenant comme complémentaires, ce qui est nouveau.

Rappelons les débats sur la place d'une approche qualitative (longtemps décriée et critiquée par le courant dominant en psychologie) et sur la place d'une approche 
expérimentale et quantitative (considérée comme étant la seule pertinente pour une analyse objective des phénomènes et des processus psychologiques). Là, encore, nous retrouvons la fameuse emprise épistémologique de la psychologie sur la psychologie de la religion qui en a subi les conséquences à l'instar des autres sous-disciplines de la psychologie. Voici ces deux orientations en psychologie de la religion :

1. La première incite à travailler sur un ensemble de processus transversaux d'inspiration psychosociale et cognitive, tels l'orthodoxie, les processus idéologiques, le dogmatisme, l'attribution, la vitesse du jugement, la mémoire. La cognition sociale serait un domaine prometteur pour la psychologie de la religion. Cette orientation s'accompagne d'une réaffirmation quant à la nécessité des méthodes expérimentales censées diminuer le poids de l'implication idéologique des chercheurs (Deconchy, 1987 ; Gorsuch, 1988 ; Hunsberger, 1991).

2. La deuxième orientation prône une démarche herméneutique de compréhension du sens produit par l'individu (Belzen \& Hood, 2006). La démarche positiviste de réduction de la religion à une fonction intra-individuelle - jugée destructrice pour le domaine de la psychologie de la religion est critiquée. Plusieurs perspectives y sont proposées : la psychologie narrative, le constructionnisme, la phénoménologie, l'approche clinique (Day, 2001 ; Gergen, 1993 ; Nørager, 1996 ; Rizzuto, 2001 ; Vande Kemp, 1999 ; Vergote, 1993) et la psychologie culturelle (Belzen, 1999, 2005b ; Vergote, 1993). Ces approches analysent la croyance en tant que discours inscrit dans des relations interpersonnelles et des contextes précis. On s'intéresse à l'inscription sociale et culturelle des phénomènes qui sont alors envisagés dans leur multidimensionnalité et pluralité culturelle. Les méthodes qualitatives et l'interdisciplinarité sont mises en avant (Aten \& Hernandez, 2005 ; Belzen \& Hood, 2006 ; Vergote, 1993) ; ces méthodes sont d'ailleurs mieux considérées qu'il y a quelques années en psychologie, comme en psychologie de la religion.

Malgré l'incompatibilité apparente initiale des deux orientations, soulignons ici l'intérêt croissant des chercheurs pour l'étude du fait religieux à de multiples niveaux d'analyse (voir par exemple: Belzen \& Hood, 2006 ; Emmons \& Paloutzian, 2003 ; Hill, 1999 ; McCrae, 1999 ; McFarland, 1984 ; Nørager, 1996 ; Van der Lans, 1977 ; 1985 ; Zinnbauer et al., 1999). Actuellement, les chercheurs s'ouvrent à la complémentarité de ces deux orientations.

Cela revient à reconnaître la complexité du fait religieux, l'importance de l'envisager dans son inscription sociale et culturelle, ainsi que le besoin d'une démarche pluriméthodologique, interdisciplinaire, faisant appel à une multitude de théories existantes. Cette évolution semble annoncer un changement paradigmatique dans le domaine de la psychologie de la religion pour les prochaines années.

Magdalini DARGENTAS

Centre de Recherches en Psychologie, Cognition et Communication (CRPCC - EA 1285), Université de Rennes 2 - Université de Bretagne occidentale. Centre Edgar Morin - IIAC (UMR 8177), École des Hautes Études en Sciences sociales) magdalini.dargentas@univ-brest.fr 


\section{Bibliographie}

Aletti Mario, 2006, "Neurobiology and Psychology of Religion: the Part and the Whole", Paper presented at the Conference of 2006, International Association for the Psychology of Religion, Leuven, Belgium.

AllPORT Gordon W., 1969, The Person in Psychology: Selected Essays (2 ${ }^{\text {nd }}$ ed.), Boston, Beacon Press.

AllPort Gordon W., 1971, Personality: A Psychological Interpretation (3 ${ }^{\text {rd }}$ ed.), London, Constable.

Allport Gordon W., Ross Michael J., 1967, "Personal Religious Orientation and Prejudice”, Journal of Personality and Social Psychology, 5, p. 432-443.

Altemeyer Bob, Hunsberger Bruce, 1992, “Authoritarianism, Religious Fundamentalism, Quest, and Prejudice", International Journal for the Psychology of Religion, 2, p. 113-133.

ARgYle Michael, 1985, "New Directions in the Psychology of Religion”, in Brown L. B. (éd.), Advances in the Psychology of Religion, Oxford, Pergamon Press, p. 8-17.

ARgyle Michael, 1988, Bodily Communication (2 ${ }^{\text {nd }}$ ed.), London, Methuen \& Co Ltd.

ARgYLE Michael, 2002, "State of the Art. Religion", The Psychologist, 15-1, p. 22-26.

Aten Jamie D., Hernandez Barbara C., 2005, “A 25-year Review of Qualitative Research published in Spiritually and Psychologically oriented Journals”, Journal of Psychology and Christianity, 24-3, p. 266-277.

BATSON Daniel C., 1979, "Experimentation in Psychology of Religion: Living With or In a Dream?”, Journal for the Scientific Study of Religion, 18-1, p. 90-93.

Batson Daniel C., Schoenrade Patricia A., Pych Virginia, 1985, "Brotherly Love or Selfconcern? Behavioural Consequences of Religion", in Brown L. B. (éd.), Advances in the Psychology of Religion, Oxford, Pergamon Press, p. 185-208.

BECK Richard, MiLler Jonathan P., 2001, "Erosion of Belief and Disbelief: Effects of Religiosity and Negative Affect on Beliefs in the Paranormal and Supernatural", The Journal of Social Psychology, 141-2, p. 277-287.

Beit-Hallahmi Benjamin, 1985, "Religiously based Differences in Approach to the Psychology of Religion: Freud, Fromm, Allport and Zilboorg”, in Brown L. B. (éd.), Advances in the Psychology of Religion, Oxford, Pergamon Press, p. 18-33.

Beit-Hallahmi Benjamin, Argyle Michael, 1997, Religious Behaviour, Belief and Experience, London, Routledge.

Belzen Jacob A., 1999, “The Cultural Psychological Approach to Religion. Contemporary Debates on the Object of the Discipline", Theory and Psychology, 9-2, p. 229-255.

Belzen Jacob A., 2005a, "In Defense of the Object: On Trends and Directions in Psychology of Religion", The International Journal for the Psychology of Religion, 15-1, p. 1-16.

Belzen Jacob A., 2005b, “A Way Out of the Crisis? From Völkerpsychologie to Cultural Psychology of Religion", Theory \& Psychology, 15-6, p. 812-838.

Belzen Jacob A., Hood Ralph W., 2006, "Methodological Issues in the Psychology of Religion: Toward Another Paradigm?”, The Journal of Psychology, 140-1, p. 5-28.

BOYER Pascal, 2003, "Religious Thought and Behaviour as By-Products of Brain Function”, TRENDS in Cognitive Sciences, 7-3, p. 119-124.

BRown Laurence B., 1985, "Social Attitudes and Religion", in Brown L. B. (éd.), Advances in the Psychology of Religion, Oxford, Pergamon Press, p. 151-170. 
BuXant Coralie, Saroglou Vassilis, Casalfiore Stefania, Christians Louis-Léon, “Cognitive and Emotional Characteristics of New Religious Movement Members: New Questions and Data on the Mental Health Issue", Mental Health, Religion \& Culture, 10-3, p. 219-238.

ChAmpion Françoise, 2013, "Introduction. Des relations entre "religieux" et "psy" ", Archives de sciences sociales des religions, 163, p. 9-16.

Clayton Richard R., Gladden James W., 1974, “The Five Dimensions of Religiosity: Toward Demythologizing a Sacred Artifact", Journal for the Scientific Study of Religion, 13-2, p. 135-143.

DARGENTAS Magdalini, 2016 sous presse, «La psychologie de la religion : dénominations, historique et controverses ", Bulletin de Psychologie.

DARGENTAS Magdalini, 2005, Le rapport à la mort et l'incinération: représentations sociales, pratiques et appartenances religieuses. Une étude auprès d'Orthodoxes et de Catholiques Grecs, Thèse de doctorat en psychologie sociale, Paris, École des Hautes Études en Sciences Sociales.

Dargentas Magdalini, Fraïssé Christèle, 2011, "Contribution à l'étude psychosociale du religieux : analyse des contenus associés au mariage homosexuel et à la crémation ", Bulletin de Psychologie, 64-2, p. 133-147.

DaY James M., 2001, "From Structuralism to Eternity? Re-Imagining the Psychology of Religious Development after the Cognitive-Developmental Paradigm", The International Journal for the Psychology of Religion, 11-3, p. 173-183.

DeConchy Jean-Pierre, 1967, Structure génétique de l'idée de Dieu chez les catholiques français. Garçons et filles de 8 à 16 ans, Bruxelles, Éditions de Lumen Vitae.

Deconchy Jean-Pierre, 1971, L'Orthodoxie Religieuse. Essai de logique psychosociale, Paris, Éditions Ouvrières.

Deconchy Jean-Pierre, 1985, "Non-experimental and Experimental Methods in the Psychology of Religion: a few Thoughts on their Implications and Limits", in Brown L. B. (éd.), Advances in the Psychology of Religion, Oxford, Pergamon Press, p. 76-112.

Deconchy Jean-Pierre, 1987, "Théories et Allégories en Psychologie de la Religion ", Archives en Sciences Sociales des Religions, 64-2, p. 179-192.

DeConchy Jean-Pierre, 1995, "Contrôle social et rationalité dans les systèmes "orthodoxes” ", in Mugny G., Oberlé D., Beauvois J.-L. (éds.), Relations humaines, groupes et influence sociale, Grenoble, PUG, p. 91-100.

DeCONCHY Jean-Pierre, 1998, «La construction mentale de la spécificité humaine : parcours expérimental dans le champ des croyances ", in Beauvois J.-L., Joule R. V., Monteil J. M. (éds.), 20 ans de psychologie sociale expérimentale francophone, Grenoble, PUG, p. 111-150.

DitTeS James E., 1969, "Psychology of Religion”, in Lindzey G., Aronson E. (éds.), The Handbook of social psychology, vol. 5, London, Addison-Wesley, p. 602-659.

Donahue Michael J., 1985, "Intrinsic and Extrinsic Religiousness: Review and MetaAnalysis", Journal of Personality and Social Psychology, 48-2, p. 400-419.

Emmons Robert A., Paloutzian Raymond F., 2003, “The Psychology of Religion”, Annual Review of Psychology, 54, p. 377-402.

Festinger Leon, RieCKen Hank, SCHACHTER Stanley, 1993, L'échec d'une prophétie (When Prophecy fails. A Social and Psychological Study of a Modern Group that Predicted the Destruction of the World, 1956), Paris, PUF. 
Fontaine Johnny R. J., Duriez Bart, LuYTen Patrick, HuTsebaut Dirk, 2003, “The internal structure of the Post-Critical Belief scale", Personality and Individual Differences, 35-3, p. 501-518.

Fowler James, 1994, "Moral Stages and the Development of Faith", in Puka B. (éd.), Fundamental Research in Moral Development, New York, Garland Publishing, p. 344-374.

Francis Leslie J., 1985, "Personality and Religion: Theory and Measurement”, in Brown L. B. (éd.), Advances in the Psychology of Religion, Oxford, Pergamon Press, p. 171-184.

GERGEN Kenneth J., 1993, "Belief as Relational Resource”, The International Journal for the Psychology of Religion, 3-4, p. 231-235.

Gorsuch Richard L., 1988, "Psychology of Religion”, Annual Review of Psychology, 39, p. 201-221.

Gorsuch Richard L., Aleshire Daniel, 1974, "Christian Faith and Ethnic Prejudice: a Review and Interpretation of Research", Journal for the Scientific Study of Religion, 13-3, p. 281-307.

HAY David, 1979, "Religious Experience Amongst a Group of Post-Graduate Students A Qualitative Study”, Journal for the Scientific Study of Religion, 18-2, p. 164-182.

HaY David, 1985, "Religious Experience and its Induction”, in Brown L. B. (éd.), Advances in the Psychology of Religion, Oxford, Pergamon Press, p. 135-150.

Hill Peter C., 1999, "Giving Religion Away: What the Study of Religion Offers Psychology", The International Journal for the Psychology of Religion, 9-4, p. 229-249.

Hood Ralph W., 1980, "Social Legitimacy, Dogmatism, and the Evaluation of Intense Experiences”, Review of Religious Research, 21-2, p. 184-194.

Huber Stefan, 2006, “The 'Structure-of-Religiosity-Test' (S-R-T), A Comprehensive Instrument for Systematic Research in the Field of Religion", Book of Abstracts, Conference of 2006, International Association for the Psychology of Religion, Leuven, Belgium, p. 55-56.

HunsBerger Bruce, 1980, “A Reexamination of the Antecedents of Apostasy”, Review of Religious Research, 21-2, p. 158-170.

HunsBerger Bruce, 1991, "Empirical Work in the Psychology of Religion”, Canadian Psychology / Psychologie Canadienne, 32-3, p. 497-507.

Hunt Richard A., 1993, "Response to Northover and Gonzalez”, The International Journal for the Psychology of Religion, 3-3, p. 201-204.

JACKSON Lynne M., ESSES Victoria M., 1997, "Of Scripture and Ascription: the Relation between Religious Fundamentalism and Intergroup Helping”, Personality and Social Psychology Bulletin, 23-8, p. 893-906.

Jodelet Denise, 1989, Folies et représentations sociales, Paris, PUF.

KirKPATRICK Lee A., 1999, “Toward an Evolutionary Psychology of Religion and Personality”, Journal of Personality, 67-6, p. 921-952.

MCCrae Robert R., 1999, "Mainstream Personality Psychology and the Study of Religion”, Journal of Personality, 67-6, p. 1209-1218.

MCFarland Sam, 1984, "Psychology of Religion: A Call for a Broader Paradigm", American Psychologist, 39-3, p. 321-324. 
Newberg Andrew, D’Aquili Eugene G., 2000, “The Neuropsychology of Religious and Spiritual Experience”, Journal of Consciousness Studies, 7, p. 251-266.

Nørager Troels, 1996, "Metapsychology and Discourse: A Note on Some Neglected Issues in the Psychology of Religion", The International Journal for the Psychology of Religion, 6-3, p. 139-149.

PeET Christopher, 2005, "Defining Religion: Strategies and Reflections on an Elusive Figure", Journal of Psychology and Christianity, 24-2, p. 105-112.

Ragan Claude, Malony Newton H., Beit-Hallahmi Benjamin, 1980, "Psychologists and Religion: Professional Factors and Personal Belief", Review of Religious Research, 21-2, p. 208-217.

RizzuTo Ana Maria, 2001, "Religious Development Beyond the Modern Paradigm Discussion: the Psychoanalytic Point of View", The International Journal for the Psychology of Religion, 11-3, p. 201-214.

Ryan Richard M., Rigby Scott, KING Kristi, 1993, “Two Types of Religious Internalization and Their Relations to Religious Orientations and Mental Health", Journal of Personality and Social Psychology, 65-3, p. 586-596.

SARoglou Vassilis, 2002, "Religion and the Five Factors of Personality: A Meta-analytic Review", Personality and Individual Differences, 32, p. 15-25.

Seligman Martin E. P., 1999, “The President's Address”, American Psychologist, 54, p. 559-562.

Streib Heinz, 2001, "Faith Development Theory Revisited: The Religious Styles Perspective", The International Journal for the Psychology of Religion, 11-3, p. 143-158.

VAN DER LANS Jan, 1977, "Religious Experience: an Argument for a Multidisciplinary Approach", The Annual Review of the Social Sciences of Religion, 1, p. 133-145.

VAn DER LANs Jan, 1985, "Frame of Reference as a Prerequisite for the Induction of Religious Experience through Meditation: an Experimental Study”, in Brown L. B. (éd.), Advances in the Psychology of Religion, Oxford, Pergamon Press, p. 127-134.

VANDE Kemp Hendrika, 1999, "Commentary on the Special Issue: Religion in the Psychology of Personality", Journal of Personality, 67-6, p. 1195-1207.

Vergote Antoine, 1993, "What the Psychology of Religion Is and What It Is Not”, The International Journal for the Psychology of Religion, 3-2, p. 73-86.

Vergote Antoine, 1996, Religion, Belief and Unbelief, Leuven, Leuven University Press.

Vergote Antoine, TAmayo Alvaro, 1981, The Parental Figures and the Representation of God. A Psychological and Cross-Cultural Study, The Hague, Mouton.

YeatTs John R., Asher William, 1979, "Can We Afford Not To Do True Experiments in Psychology of Religion? A Reply to Batson”, Journal for the Scientific Study of Religion, 18-1, p. 86-89.

Zinnbauer Brian J., Pargament Kenneth I., SCotT Allie B., 1999, “The Emerging Meanings of Religiousness and Spirituality: Problems and Prospects", Journal of Personality, 67-6, p. 889-919. 


\section{La psychologie de la religion : courants de recherche et perspectives théoriques}

Bien que la psychologie de la religion soit actuellement peu étudiée en France, son développement est important dans le monde anglo-saxon. Sont ici recensées les études autour de la religiosité et de ses corrélats, les théories sur la croyance et les études autour d'autres formes d'expériences religieuses et spirituelles. Des approches théoriques, telles que la neuropsychologie, la psychologie cognitive et sociale sont également abordées. L'état des lieux expose enfin les questionnements transversaux et les tendances théoriques et empiriques actuelles.

Mots-clés: Psychologie de la religion, religiosité, croyance, spiritualité, approches théoriques.

\section{Psychology of Religion: research trends and theoretical perspectives}

Although psychology of religion is little studied in France, it is a very developed field today in the Anglo Saxon world. In this paper will be reviewed studies on religiosity and its correlates, studies theories on belief, and studies on other forms of religious and spiritual experiences. Theoretical approaches, such as neuropsychology and cognitive and social psychology will also be addressed. This well-needed overview will offer a systematical exposure of today's transversal questions and theoretical and empirical trends.

Key words: Psychology of religion, religiosity, faith, spirituality, theoretical frameworks. 